\title{
A Comparative Study: Modified Particle Swarm Optimization and Modified Genetic Algorithm for Global Mobile Robot Navigation
}

\author{
Nadia Adnan Shiltagh \\ Computer Department \\ College of Engineering \\ University of Baghdad
}

\author{
Lana Dalawr Jalal \\ Computer and Automation Department \\ College of Engineering \\ University of Sulaimani
}

\begin{abstract}
In this paper, Modified Genetic Algorithm $\left(M G A^{* I}\right)$ and Modified Particle Swarm Optimization (MPSO*I ) are developed to increase the capability of the optimization algorithms for a global path planning which means that environment models have been known already. The proposed algorithms read the map of the environment which expressed by grid model and then creates an optimal or near optimal collision free path. The effectiveness of these optimization algorithms for mobile robot path planning is demonstrated by simulation studies. This paper investigates the application of efficient optimization algorithms, $M G A^{*}$ and $M P S O *$ to the problem of mobile robot navigation.
\end{abstract}

Despite the fact that Genetic Algorithm (GA) has rapid search and high search quality, infeasible paths and high computational cost problems are exist associated with this algorithm. To address these problems, the $M G A^{*}$ is presented. Adaptive population size without selection and mutation operators are used in the proposed algorithm. In this thesis, Distinguish Algorithm (DA) is used to check the paths, whether the path is feasible or not, in order to come out with all feasible paths in the population.

Improvements presented in $M P S O^{*}$ are mainly trying to address the problem of premature convergence associated with the original $P S O$. In the $M P S O^{*}$ an error factor is modelled to ensure that the PSO converges. MPSO* try to address another problem which is the population may contain many infeasible paths. A modified procedure is carrying out in the $M P S O$ * to solve the infeasible path problem.

According to the simulation done using MATLAB version $\mathrm{R} 2012$ (m-file), both algorithms (MGA* and $M P S O^{*}$ ) are tested in different environments and the results are compared. The results demonstrate that these two algorithms have a great potential to solve mobile robot path planning with satisfactory results in terms of minimizing distance and execution time. The simulation results illustrate that the path obtained by $M G A^{*}$ is the shortest path, however the execution time based on MPSO* is significantly smaller than the execution time of $M G A^{*}$. Thus, the proposed $M P S O *$ is computationally faster than the $M G A^{*}$ in finding optimal path.

1 The $(*)$ used to distinguish the proposed modified algorithms (MGA and MPSO) from the previous modified algorithms.

\section{Keywords}

Modified Genetic Algorithm (MGA*), Modified Particle Swarm Optimization (MPSO*), path planning, mobile robot navigation.

\section{INTRODUCTION}

A robot is a controlled manipulator capable of performing a variety of tasks and decision-making like human beings, and may be either fixed in place or mobile. Mobility is an important consideration for modern robots [1]. As new technological achievements take place in the robotic hardware field, an increased level of intelligence is required as well. The most fundamental intelligent task for a mobile robot is the ability to plan a valid path from its initial to terminal configuration while avoiding all obstacles located on its way which is known as robot path planning [2]

The efficiency of the mobile robot path planning is considered as an important matter since one of the main concerns is to find the destination in a short time. Accordingly, a desirable path should result from not letting the robot waste time taking unnecessary steps or becoming stuck in local minimum positions. Furthermore, a desirable path should avoid all known obstacles in the area [3].

There are so many methods that have been developed to overcome the path planning problem for mobile robots. Each method differs in its effectiveness depending on the type of application environment and each one of them has its own strengths and weaknesses. The choice of a good heuristic algorithm is necessary in order to achieve both quality and efficiency of a search. Generally, some optimization criteria with respect to time and distance must be satisfied. Other constraints with respect to velocity and acceleration of mobile robots should also be taken into consideration [4].

\section{RELATED WORKS}

Mohanty and Parhi, described the various techniques applied for navigation of an intelligent mobile robot. They showed that the heuristic approaches such as Fuzzy logic (FL), Artificial Neural Networks (ANN), Neuro-Fuzzy (NF), Genetic Algorithm (GA), Particle Swarm Optimization (PSO), Ant Colony Optimization (ACO) and Artificial Immune System, gave suitable and effective results for mobile robot navigation as a target reaching and obstacle-avoidance. These techniques are also helpful for the solution of the local minima problem. Each method has its own strength over the others in certain aspects, researchers have been seeking for more efficient ways to solve this problem. In this section, the recent works on robot's navigation and path planning are reviewed [5]. 
(Dutta, 2010) deal with the obstacle avoidance of a wheeled mobile robot in structured environment by using PSO based neuro-fuzzy approach, and three layer neural networks with PSO were used as learning algorithm to determine the optimal collision-free path [6].

(Tamilselvi et al., 2011) suggested navigation strategy using GA for path planning of a mobile robot. The most important modification applied to the algorithm is the fitness evaluation. The efficiency of the algorithm is analysed with respect to execution time and path cost to reach the destination. Path planning, collision avoidance and obstacle avoidance are achieved in both static and dynamic environment [7].

(Ragavan et al., 2011) presented two optimization algorithms to solve path planning problem for robot navigation. The PSO and Genetic Algorithm - Artificial Immune System (GA-AIS) were developed and implemented for the path optimization. They concluded that the PSO algorithm has much faster computational time than GA-AIS algorithm for small network, while GA-AIS shows improvement and appears to perform better for large networks [8].

(Raja and Pugazhenthi, 2012) provided an overview of the research progress in path planning of a mobile robot for offline as well as on-line environments. Commonly used classic and evolutionary approaches of path planning of mobile robots have been discussed, and they showed that the evolutionary optimization algorithms are computationally efficient. Also, challenges involved in developing a computationally efficient path planning algorithm are addressed [9].

(Ahmadzadeh and Ghanavati, 2012) presented an intelligent approach for the navigation of a mobile robot in unknown environments, the navigation problem becomes an optimization problem, and then it is solved by PSO algorithm. Based on position of goal, an evaluation function for every particle in PSO is calculated. It's assumed that robot can detect only obstacles in a limited radius of surrounding with its sensors. Environment is supposed to be dynamic and obstacles can be fixed or movable [10].

(Habeeb, 2012) presented a modified genetic algorithm and showed that it has good results in the path planning of mobile robots in terms of minimizing distance and execution time. Also they illustrated that the modified genetic algorithm leads to a very quick generation of a path solution because no mutation operation was used which leads to consuming the time [11].

(Yu-qin and Xiao-peng, 2012) provided an Immune Particle Swarm Optimization (IPSO) algorithm for path planning of the mobile robot which based on the biological mechanism of the immune system. They compared the simulation results with Dijkstra algorithm and PSO optimization results. They concluded that the optimal path and the execution time based on IPSO algorithm are reduced separately, and the improved PSO algorithm enhances the convergence speed and robustness of time-varying parameters [12].

(Gigras and Gupta, 2012) proposed the use of ACO algorithm for robot motion control such as navigation and obstacle avoidance in an efficient manner. They showed that by using this algorithm, money can be saved and reliability can be increased by allowing them to adapt themselves according to the environment without further programming [13].

From the above literature review for the recently published paper, the following points are highlighted and emphasized:

1. The simulation and experimental results from previous researches show that algorithms play an important role to produce an optimal path for mobile robot navigation.

2. Various works of research have been successfully applied $P S O$ to solve the mobile robot path planning problem due to its simplicity and efficiency in navigating large search spaces for optimal solutions.

3. Many researchers have been worked in the field of path planning using $G A$ to generate the optimal path by taking the advantage of its strong optimization capability.

4. A $G A$ is more efficient than the fuzzy logic and neural networks. This is due to the capability of genetic algorithms to optimize both discrete and continuous mappings.

\section{MODIFIED GENETIC \\ ALGORITHMS (MGA*)}

In this paper, a modified genetic algorithm is proposed based on the traditional genetic algorithm. Path planning using $M G A *$ does not require any encoding scheme because it uses the real representation which reduces calculation time. The $M G A^{*}$ starts with generation of initial population; which may contain feasible and infeasible paths. To generate the initial population and verify that the generated path in the initial population is feasible or not, Distinguish Algorithm $(D A)$ is used, and also, the $M G A^{*}$ leads to quick generation of a path solution because no selection and mutation operations are used which leads to decrease the execution time. The goal of $M G A^{*}$ is to minimize the total distance from the starting position to the desired position without colliding with any of the obstacles in the environment with satisfied time. The flowchart of the modified genetic algorithm is illustrated in Figure 1. 


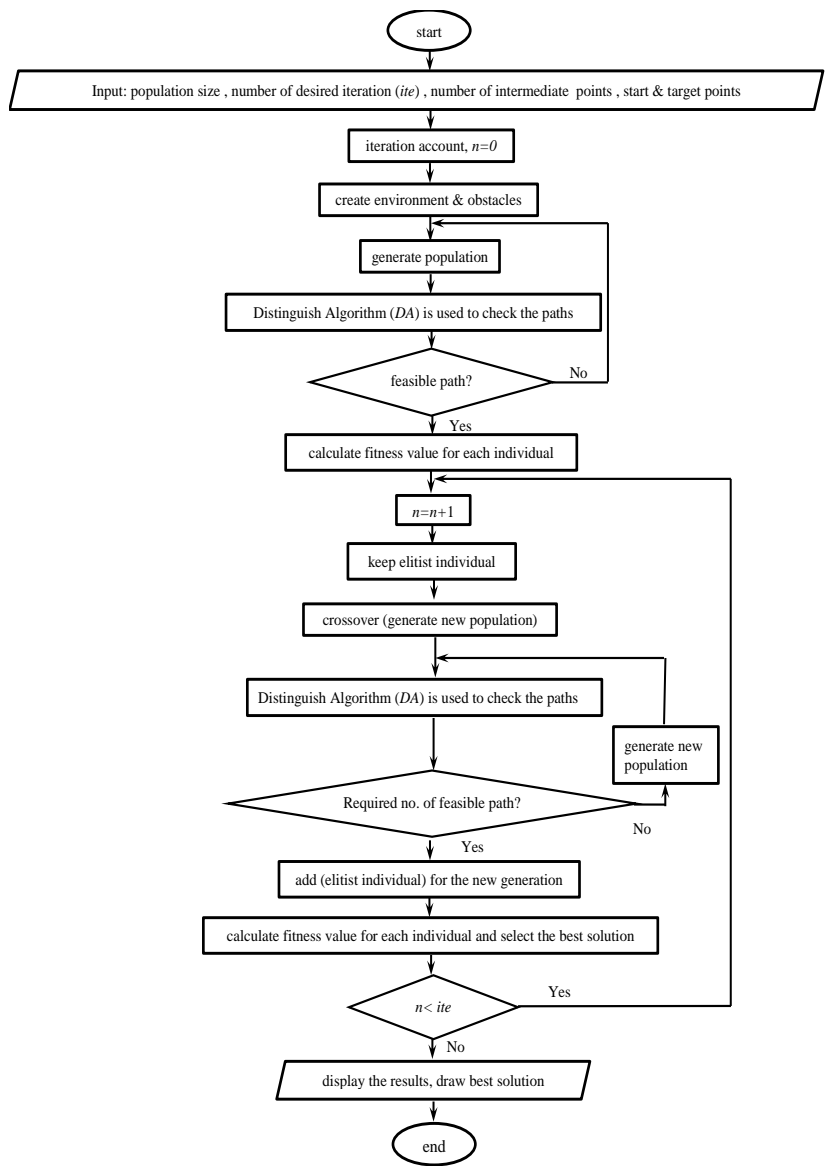

As the number of obstacles increases, the algorithm needs more generations to find the best solution. Obstacle areas in the working environment are represented by blue squares which contain red grid and has an average area of $1 \times 1$ unit. Boundaries for obstacles area are formed by their actual boundaries plus a safety distance that is defined with consideration to the size of the mobile robot which is treated as a point in the environment. In each environment the obstacles are randomly put but carefully placed in such a way that they keep some distance from the starting point to the target point to make sure that the robot has some space to move.

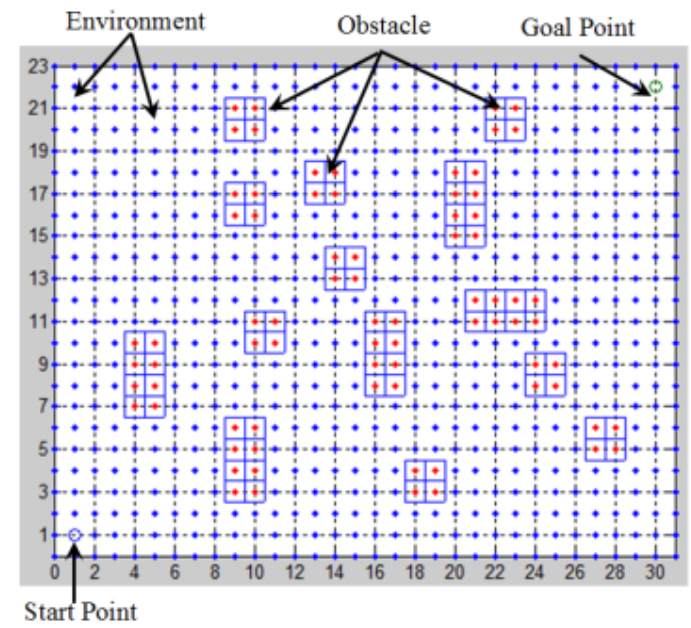

Fig 2: First working environment

Fig 1: Flowchart of the Modified Genetic Algorithm*

The process of modified genetic algorithm is illustrated in the following steps:

\subsection{Creating the Environment, and Obstacles in the Algorithm}

To verify the effectiveness of the MGA*, the simulation has been applied for different grid settings and different number of obstacles. Different known working environments are used, which are presented in Figure 2 to Figure 3. As displayed in the figures, the blue grids represent obstacle free areas where mobile robot can move freely. There is no unit used to measure the path length because each cell in the environments can represent any unit. The circle sign in the environment is the robot's starting and goal location. The grid size, the coordinate of the start and target points, and the number of obstacles for each environment are shown in Table 1.

Table 1: Grid size, start and target point, number of obstacles

\begin{tabular}{|c|c|c|c|c||}
\hline Environments & $\begin{array}{l}\text { grid size } \\
\text { [unit] }\end{array}$ & $\begin{array}{c}\text { Start } \\
\text { point } \\
\text { [unit] }\end{array}$ & $\begin{array}{c}\text { target } \\
\text { point } \\
\text { [unit] }\end{array}$ & $\begin{array}{c}\text { number } \\
\text { of } \\
\text { obstacles }\end{array}$ \\
\hline $1^{\text {st }}$ environment & $31 \times 23$ & $\begin{array}{c}\mathrm{x}=1, \\
\mathrm{y}=1\end{array}$ & $\begin{array}{c}\mathrm{x}=22, \\
\mathrm{y}=30\end{array}$ & 76 \\
\hline $2^{\text {nd }}$ environment & $20 \times 20$ & $\begin{array}{c}\mathrm{x}=1, \\
\mathrm{y}=1\end{array}$ & $\begin{array}{c}\mathrm{x}=7 \\
\mathrm{y}=6\end{array}$ & 94 \\
\hline $\begin{array}{l}\text { The } 3^{\text {rd }} \text { environment represents the two previous } \\
\text { environments which turned to closed environments. }\end{array}$ \\
\hline
\end{tabular}

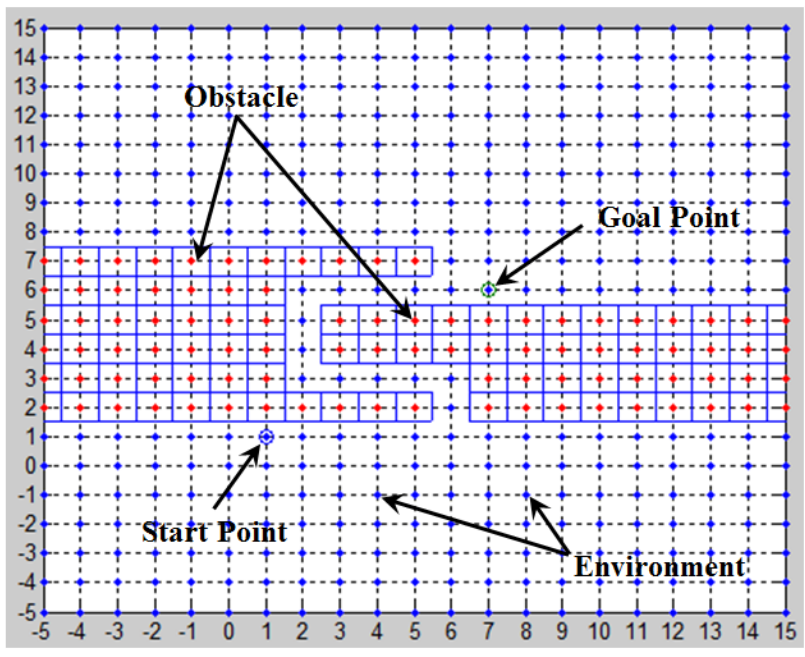

Fig 3: Second working environment

\subsection{Generating the Initial Population in the Algorithm}

All individuals of the initial population are assumed to be generated randomly. This leads to generate large numbers of infeasible paths which intersect an obstacle, and infeasible paths should be avoided. If there is/are obstacle(s) either in the vertical direction or horizontal direction, the mobile robot has to keep itself away from the obstacles.

Initial population stored in a single matrix of size $\mathrm{c} \times \mathrm{m}$, where $\mathrm{c}$ is the number of individuals in the population and $\mathrm{m}$ is the length of the individuals. Each row corresponds to a particular solution. After generating the initial population, and 
to allow the robot to move between its current and final configurations without any collision within the surrounding environment, each individual must be checked whether it intersects an obstacle or not, as explained in the next step.

\subsection{Distinguish Algorithm (DA)}

Because there are both feasible path and infeasible path in the population, the Distinguish Algorithm (DA) is used to check the paths, whether the path is feasible or not, in order to come out with all feasible individuals in the population. After applying this algorithm, feasible path will be used for next generation and infeasible path will be deleted.

The details of the $D A$ are as follows [4]:

a. Generate the line equation of $a\left(x_{1}, y_{1}\right)$ and $b\left(x_{2}, y_{2}\right)$ by:

$$
\left(y-y_{1}\right) /\left(x-x_{1}\right)=\left(y_{2}-y_{1}\right) /\left(x_{2}-x_{1}\right)
$$

$$
y=\left(x-x_{1}\right)\left(y_{2}-y_{1}\right) /\left(x_{2}-x_{1}\right)+y_{1}
$$

Then, find the intermediate points between point $a$ to point $b$, when the value of $x$ is increase with 0.5 . For each intermediate point, substitute the corresponding $x$ value in equation $3-2$ and calculate the $y$ value.

b. Check if the coordinate of the intermediate point lies within the obstacle area or obstacle free area by checking the particular intermediate point and obstacle list.

c. If the particular intermediate point lies within obstacle free area, repeat Step 2 until all intermediate points between point $a$ and point $b$ are explored. In case where any of the intermediate points lies inside obstacle area, $D A$ is terminated and that particular path will be categorized as infeasible path.

d. If none of the intermediate points lie in the obstacle area, Step 1 to Step 3 are repeated until all points in the particular path are explored. This explored path is categorized as feasible path.

\subsection{Fitness Function}

The length of the feasible path is compute as:

$$
d_{(i)}=\sqrt{\left(x_{i+1}-x_{i}\right)^{2}+\left(y_{i+1}-y_{i}\right)^{2}}
$$

$d_{(i)}$ is the distance between two points, $x_{i}$ and $y_{i}$ are robot's current horizontal and vertical positions, $x_{i+1}$ and $y_{i+1}$ are robot's next horizontal and vertical positions. The objective function of the overall path can be expressed as:

$$
\operatorname{dist}_{(j)}=\sum_{i=1}^{h} d_{(i)}
$$

where $\operatorname{dist}_{(j)}$ is the distance of the $j^{\text {th }}$ path in the environment, and $h$ is the number of links that consist the path.

The fitness function is the inverse of the total distance which is Euclidean distance. The Euclidean distance between starting and target point is the length of the line segment connecting them. The fitness function used in this thesis is (Yun et al., 2010):

$$
F_{(j)}=1 / \operatorname{dist}_{(j)}
$$

where $F_{(j)}$ is the fitness function and $j$ represents the $j^{\text {th }}$ path. It is obvious that the best individual will have the maximum fitness value. At each generation, iteration, all the chromosomes will be updated by their fitness.

\subsection{Elitism}

In order to keep the best chromosome from each generation, the elitism method is employed. In elitism it is obvious that the best individual will have maximum fitness value. The main goal of the elitism rule is to keep the best chromosome from the current generation. Thus, under this rule, the best chromosome from each generation will not undergo any mutation or crossover event and will safely move to the next generation. Since the best or elite member between generations is never lost, the performance of GA can significantly be improved.

The remaining chromosomes are then sorted according to their fitness. Since small population sizes lose diversity very fast, therefore in the proposed algorithm no selection operator is used, and all the remaining chromosomes will be selected to undergo the crossover operator. Using this approach will increase the expectation of maintaining diversity in the population.

\subsection{Crossover Operator}

After selection of the elite member, crossover operator stage starts. In crossover a group of chromosomes undergoes crossover at each generation. All the crossover events are controlled by a certain crossover probability $P_{c}$, which is equal to 0.5 . The algorithm creates a random number in range [0 1] for each chromosome. If the generated number is less than $P c$, the chromosome is a candidate for the crossover event, otherwise the chromosome proceed without crossover. The left most genes and the right most genes will avoid the crossover event since these two points are the start and target points and cannot be eliminated. For the purpose of diversity, the crossover point is randomly selected in each generation. Single-point crossover operator is used in proposed algorithm. The genes of two parents' individuals before or after the crossover point are swapped, then the individual of the father replaced by individual of the offspring. Finally, the $D A$ is used to check the paths of the newly generated offsprings. There is no mutation operator in the proposed $M G A^{*}$. After crossover operator has been applied to the initial population, a new population will be formed.

\subsection{Generation Algorithm}

Commonly in the complex environment along with high density of obstructions the number of generated paths is inefficient. Generation algorithm is used to increase the population and help to prevent the stagnating at local optima, and then the new generated population must be checked for infeasibility. If the number of generated paths is still insufficient a new population is generated. This process is repeated over again until the desired number of feasible path is reached. So, the proposed method will not use fixed population size but adaptive population size. Generated population formed in this way increase the efficiency of the proposed algorithm, and will not lose the overall genetic algorithm searching capabilities. In the next iteration, generation algorithm generates paths continuously instead of infeasible paths. 
Genetic algorithm is terminated when the maximum number of iteration exceeds a certain limit, and also terminated when it does not find a path from the start point to the target point.

\section{MODIFIED PARTICLE SWARM OPTIMIZATION (MPSO*)}

Basically the $M P S O^{*}$, just like the $P S O$, consists of a population of particles that collectively search in the search space for the global optimum. Improvements are mainly trying to address the problem of premature convergence associated with the standard PSO. These improvements usually try to solve this problem by increasing the diversity of solutions in the swarm. In the MPSO* an error factor is modelled to ensure that the $P S O$ converges. The MPSO* tries to address another problem which is population may include many infeasible paths which have undesirable effect on the performance of the algorithm. In the MPSO*, the infeasible paths are not discarded but can be modified to be feasible path. For example, if the particle path falls with an obstacle boundary, it is relocated to a position outside the obstacle. The process of $M P S O^{*}$ is demonstrated in the following subsections

\subsection{Modeling the Environment, and Obstacles in the Algorithm}

The flowchart of the proposed $M P S O *$ is illustrated in Figure 4.

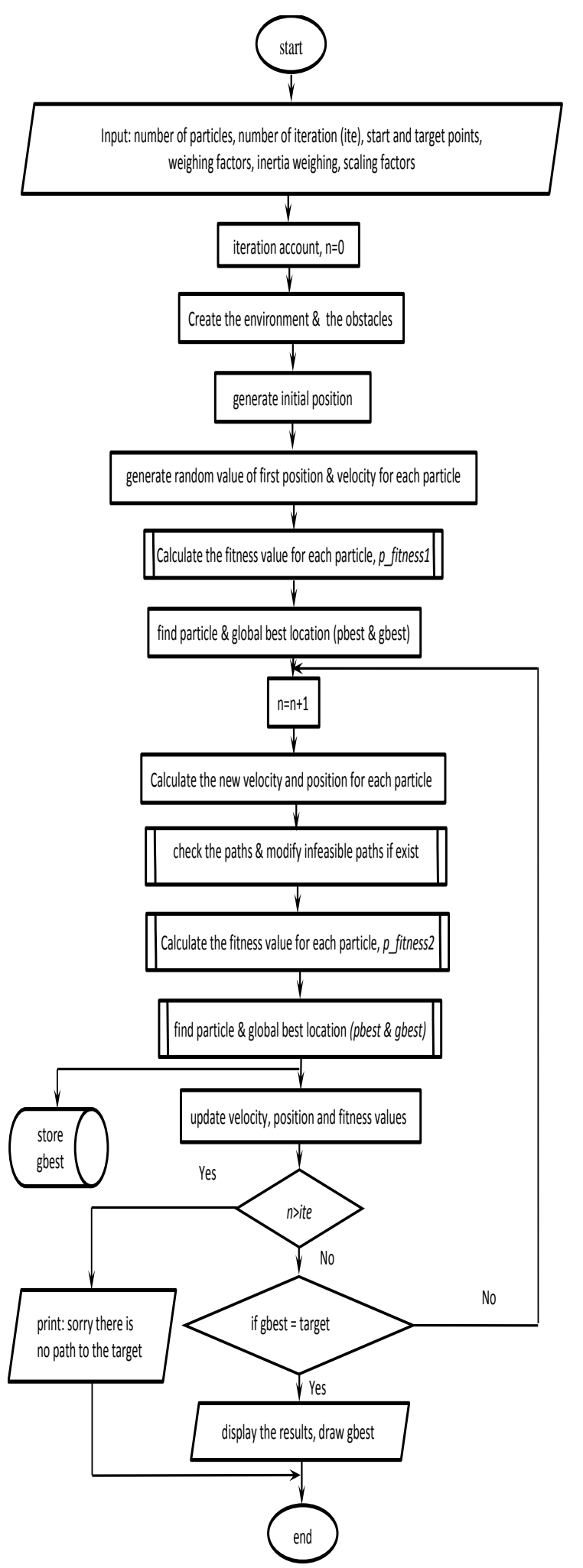

Fig 4: Flowchart of the Modified Particle Swarm Optimization

\subsection{Particle swarm initialization}

The starting position for each particle is the starting point of the path, then the first position and velocity of each particle is generated randomly but limited to the boundaries of the search space. The particle's position stored in a matrix with size of $2 \times 1$, where 1 is the number of particles. The first and 
second row of the matrix correspond the $\mathrm{x}$ and $\mathrm{y}$-coordinate for all particles, respectively.

\subsection{Fitness Function Evaluation}

The particle's paths are evaluated during each step. Evaluation function is used to provide a measure of how particles have performed in the problem domain. In the MPSO*, two parameters, the distance between points and travel time, indicated by the particles are used to calculate the particle fitness (p_fitness). Since the fitness should increase as the distance and time decrease, the fitness function for each particle of a feasible path is turned out to be a minimization problem and it is evaluated as:

$$
\begin{gathered}
p_{-} \text {fitness }= \\
1 /\left(w_{d} * \mathbf{d}\left(p_{i}, p_{i+1}\right)+w_{t} * \mathbf{t}\left(p_{i}, p_{i+1}\right)\right)
\end{gathered}
$$

where $w_{d}$ and $w_{t}$ are weighing factor for distance and travel time respectively, $d\left(p_{i}, p_{i+1}\right)$ denotes Euclidean distance between point $p_{i}$ to the next point $p_{i+1}$ for the same particle and $t\left(p_{i}, p_{i+1}\right)$ denotes time taken by the particle to cover from $p_{i}$ to $p_{i+1}$ :

$$
\begin{aligned}
& \mathbf{d}_{\left(p_{i}, p_{i+1}\right)}=\sqrt{\left(x_{i+1}-x_{i}\right)^{2}+\left(y_{i+1}-y_{i}\right)^{2}} \\
& \mathbf{t}_{\left(p_{i}, p_{i+1}\right)}=\mathbf{d}_{\left(p_{i}, p_{i+1}\right)} / v_{i}
\end{aligned}
$$

where $x_{i}$ and $y_{i}$ are particle's current horizontal and vertical positions, $x_{i+1}$ and $y_{i+1}$ are particle's next horizontal and vertical positions, and $v_{i}$ denotes the velocity of the particle when traveling from $p_{i}$ to $p_{i+1}$. The calculation of the fitness value is illustrated by the flowchart shown in Figure 5 .

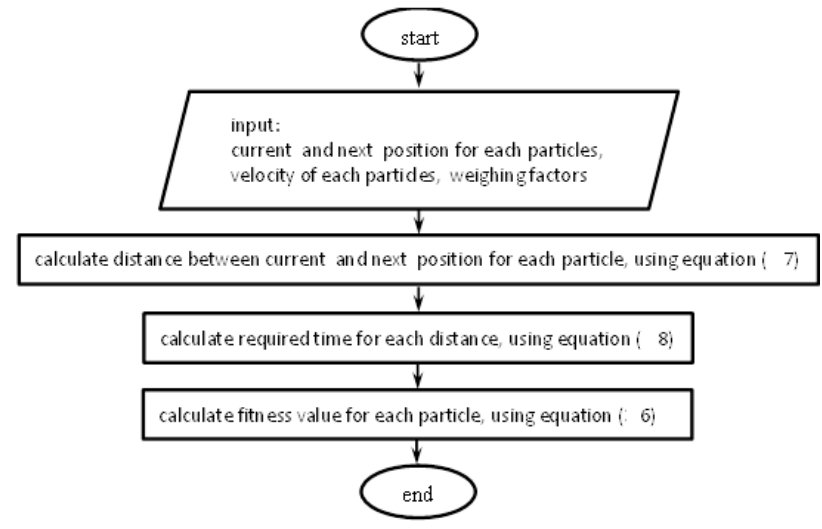

Fig 5: Flowchart for fitness calculation

\subsection{Particle position and velocity update}

The MPSO* algorithm is initialized with a number of particles and then searches for optimal. The position of a particle is influenced by the best position visited by itself which is referred to as particle best "pbest", and the best position in the whole swarm which is referred to as a global best "gbest". A particle updates its position and velocity using the following equations [14]:

$$
\begin{aligned}
& v x_{2}=w * v x_{1}+c_{1} * r_{1} *\left(p b e s t-x_{1}\right)+c_{2} * \\
& r_{2} *\left(g b e s t-x_{1}\right) \\
& v y_{2}=w * v y_{1}+c_{1} * r_{1} *\left(p b e s t-y_{1}\right)+c_{2} * \\
& r_{2} *\left(g b e s t-y_{1}\right)
\end{aligned}
$$

where the cognitive component, (pbest $\left.-x_{1}\right)$, represents the particle's own experience as to where the best solution is and the social component, $\left(\right.$ gbest $\left.-x_{1}\right)$ represents the belief of the entire swarm as to where the best solution is. Both $v x_{2}$ and $v y_{2}$ are the updated particle's velocity, $v x_{1}, v y_{1}, \mathrm{x} 1$ and $\mathrm{y} 1$ are current particle's velocity and position along $\mathrm{x}$ and $\mathrm{y}-$ direction respectively. Updating velocities is the way that enables the particle to search around its individual best position and global best position.

Self-confidence factor, $c_{1}$ and swarm-confidence factor, $c_{2}$ makes particles have the function of self-summary and learn to the best of the swarm, and get close to the best position of its own as well as within the swarm.

The inertia weight $(w)$ was first introduced by [15]. This term serves as a memory of previous velocities and it employed to control the impact of the previous velocity on the current velocity .The value of inertia factor $(w)$ is in the range of $[0$ 1]. $r_{1}$ and $r_{2}$ are random numbers within the interval of [0 1 ]. These parameters have considerable effects on performance of PSO.

In the basic concept of $P S O$ algorithm no error model is used for updating the position. However, in the MPSO* these errors are modelled to ensure that the $P S O$ converges, the modified position update equation for each particle with the error is determined as follow:

$$
\begin{aligned}
& x_{2}=x_{1}+v x_{2}+r * \text { Error } x \\
& y_{2}=y_{1}+v y_{2}+r * \text { Errory }
\end{aligned}
$$

where $x_{2}$ and $y_{2}$ are the updated positions along $x$ and $y$ direction, $r$ is a random number, large values of this parameter leads to global search, while small values leads to fine and local search which is suitable when algorithm converges. Errorx and Errory are position error along $x$ and $y$-coordinate for all particles, respectively, and it's between current particle position and the target position. These errors are used to change the particle's direction to head toward the target.

$$
\begin{aligned}
& \text { Errorx }=x_{p}-x_{t} \\
& \text { Errory }=y_{p}-y_{t}
\end{aligned}
$$

The error value is determined for each particle where $x_{p} \& y_{p}$ are the current coordinates of the individual particle and $x_{t} \&$ $y_{t}$ are the target coordinates. Based on the above equations, the next possible velocity and position of each particle is determined. If the next possible position resides within the obstacle space, the obstacle avoidance part of the algorithm is employed and the Distinguish Algorithm (DA) is used to check the paths.

\subsection{Generation of Feasible Paths}

As the particles move through the search space from current position to new position, a conditional statement is used to check all the particles paths to see if the particles are passing through obstacles. If this condition is true, the path is considered to be infeasible and the obstacle avoidance section of the algorithm is initiated. If particle path does not interfere with the position of the obstacle, the path is considered to be feasible. In the $M P S O^{*}$, the infeasible paths are not discarded but can be modified in a way that the selected path would be a collision free by using the proposed algorithm, which is 
illustrated by the flowchart in Figure 6. As shown in the figure, if the particle path falls with an obstacle boundary, it is relocated to a position outside of the obstacle.

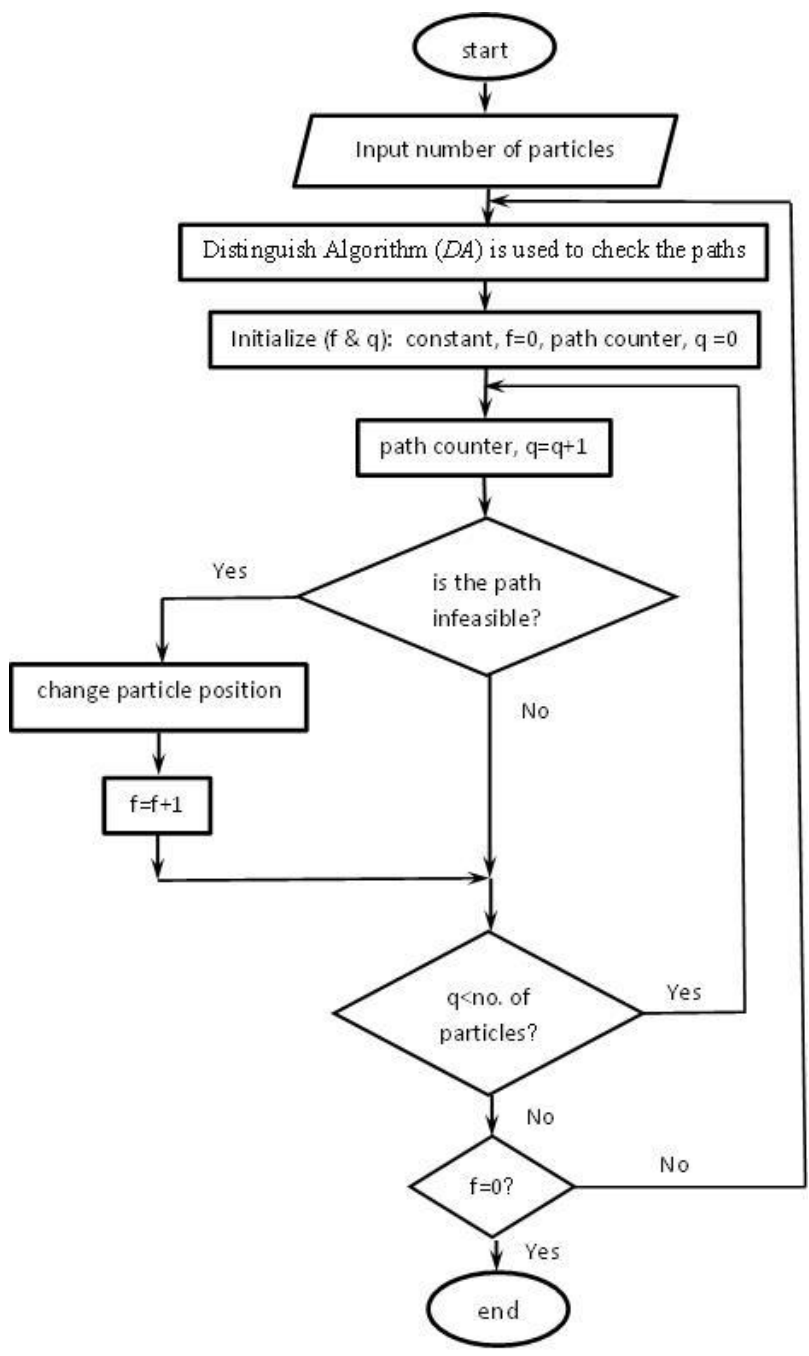

Fig 6: Flowchart for modifying the infeasible paths position

As the next step, the particle's fitness value is compared between current and new location at each iteration to determine the best position pbest for each particle, and the particle with the best fitness value is assigned as the global best $g$ best, as illustrated by the flowchart shown in Figure 7.

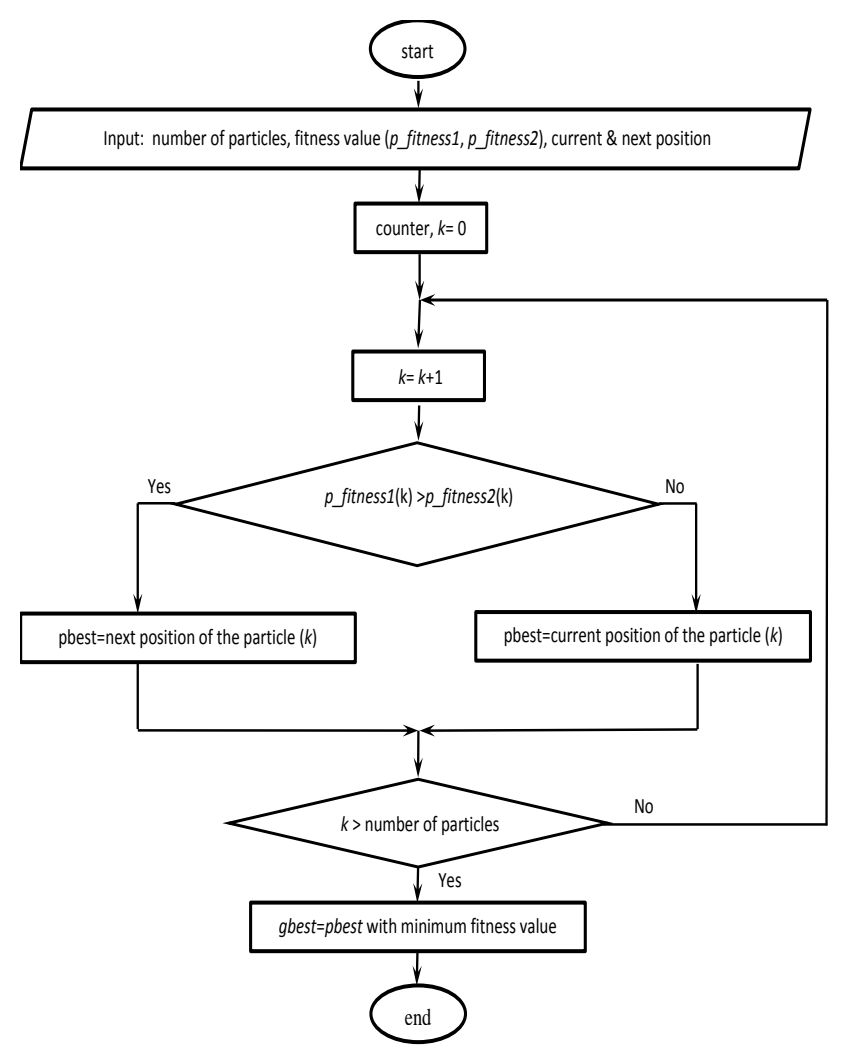

Fig 7: Flowchart for finding the particle and global best location

The MPSO* algorithm has a main nested loop terminated when the total number of iterations exceeds a certain limit or solution convergence; otherwise the program is terminated when the program could not find the path from start position to the target position. The path generated by $M P S O^{*}$ is a group of "gbest" points, including start and target points. In all cases, an optimal path is formed by line segment which is connecting the global best position "gbest" falling on the grids of the working environment.

\section{SIMULATION RESULTS}

The simulation results of mobile robot path planning using Modified Genetic Algorithm $\left(M G A^{*}\right)$ and Modified Particle Swarm Optimization $\left(M P S O^{*}\right)$ are presented to find the optimal path along the obstacle-free directions. To illustrate its wide applicability and their effectiveness, the proposed algorithms are implemented to solve the path planning problem through the computer simulation for different working environments. For comparison and validation purpose, the results obtained from the $M P S O^{*}$ algorithm are compared with results obtained by $M G A^{*}$. The programs are written by MATLAB R2012a and run on a computer with 2.5 $\mathrm{GHz}$ Intel Core i5 and $6 \mathrm{~GB} R A M$.

\subsection{The Implementation of Modified Genetic Algorithm $(M G A *)$ in Path Planning}

The implementation of the $M G A^{*}$ to solve path planning problem is demonstrated to different types of working environments. These environments are large in size and complex which have been presented to test the performance of this algorithm to find the optimal or near optimal path with satisfied time. For the proposed algorithm the simulation parameters are set as: population size $=100$, crossover probability $\left(P_{c}=0.5\right)$. In all cases, an optimal path is formed 
by line segments which are connecting the points falling on the grids of the working environment.

\subsubsection{Implementation of $M G A *$ for the First}

Environment

The performance of the $M G A^{*}$ is tested by applying this algorithm to find the optimal or near optimal path for the $1^{\text {st }}$ environment shown in Figure (2). As illustrated in the figure, this working environment is a very complex environment. The best results obtained from the implementation of the $M G A^{*}$ are shown in table 1, and the best path found is illustrated by Figure (7) to Figure (12).

Table 1: The simulation results for the $1^{\text {th }}$ environment using $M G A^{*}$

\begin{tabular}{|l|c|c|c||}
\hline \multicolumn{4}{|c||}{ Simulation Results } \\
\hline $\begin{array}{l}\text { Initial } \\
\text { Population }\end{array}$ & Iterations & Distance & Elapsed time [s] \\
\hline 100 & 20 & 36.6815 & 14.0 \\
\hline 100 & 10 & 36.7174 & 9.5 \\
\hline 100 & 5 & 37.9303 & 5.63 \\
\hline 100 & 3 & 38.6729 & 4.5 \\
\hline 100 & 2 & 38.9300 & 4.4 \\
\hline 100 & 1 & 39.4776 & 3.0 \\
\hline
\end{tabular}

The best solution obtained by $M G A^{*}$ shown that the elapsed time is 5.63 seconds to find shortest generated path with the length of 37.9303. However, the length of the path for 10 and 20 iterations is a bit shorter than 5 iterations but the computation time is much larger. From these results, one can concluded that, the required time to find optimal path is small.

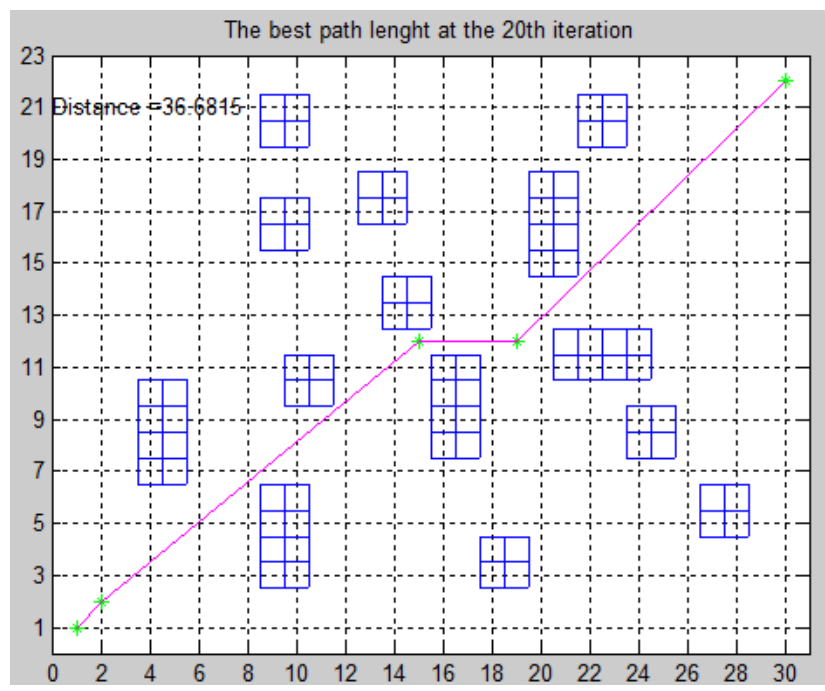

Fig 7: Path generated for the 1st environment using MGA* (20 iterations)

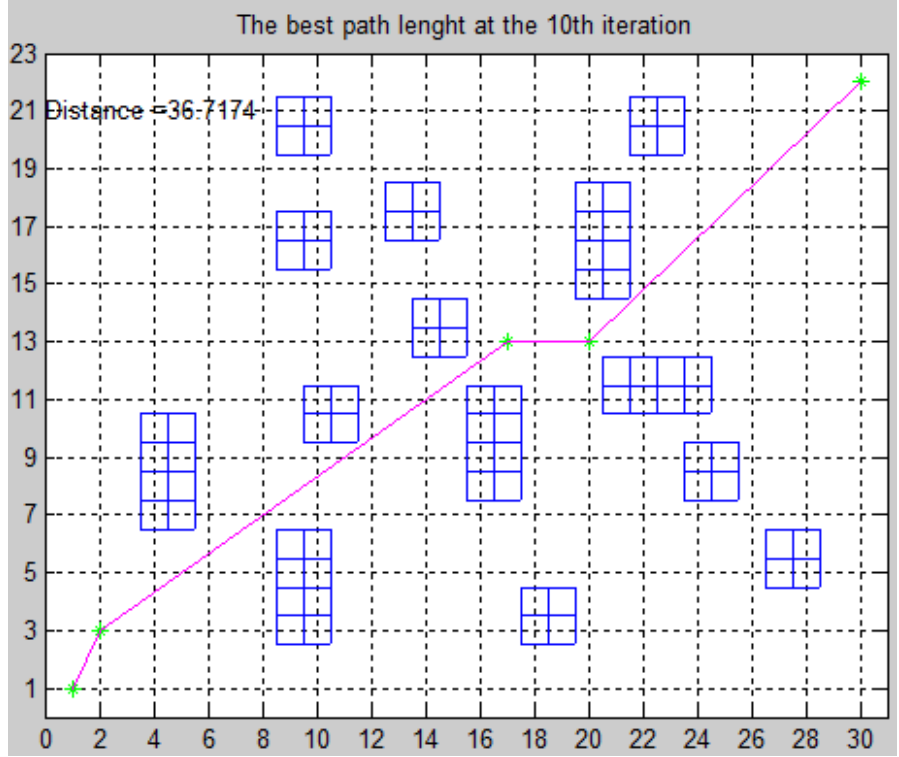

Fig 8: Path generated for the 1st environment using MGA* (10 iterations)

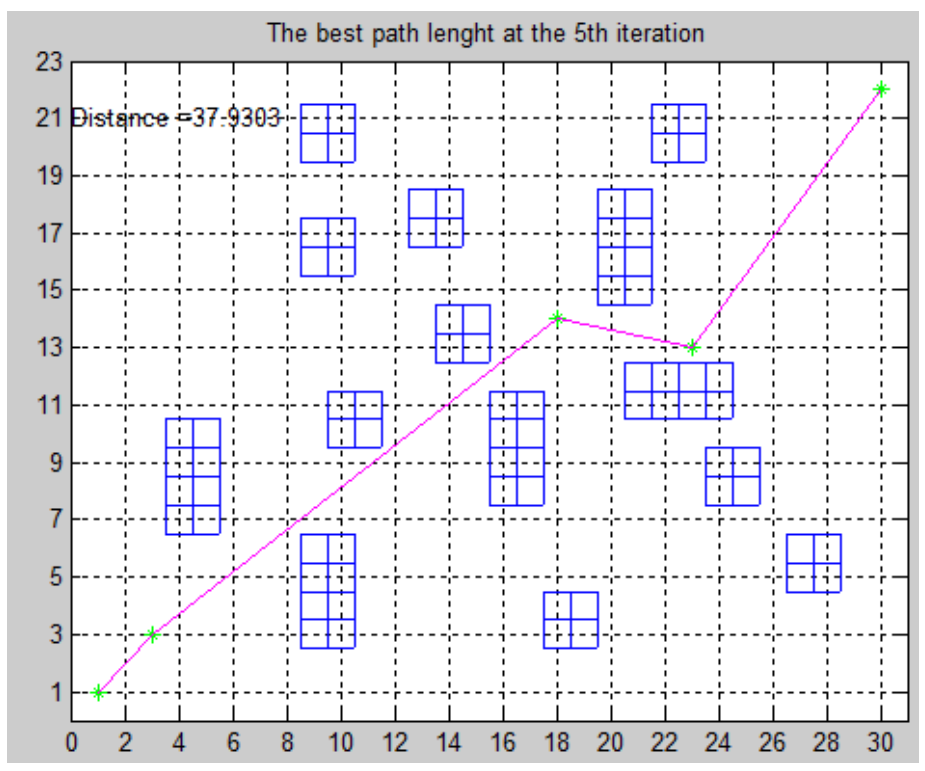

Fig 9: Path generated for the 1st environment using MGA* (5 iterations) 


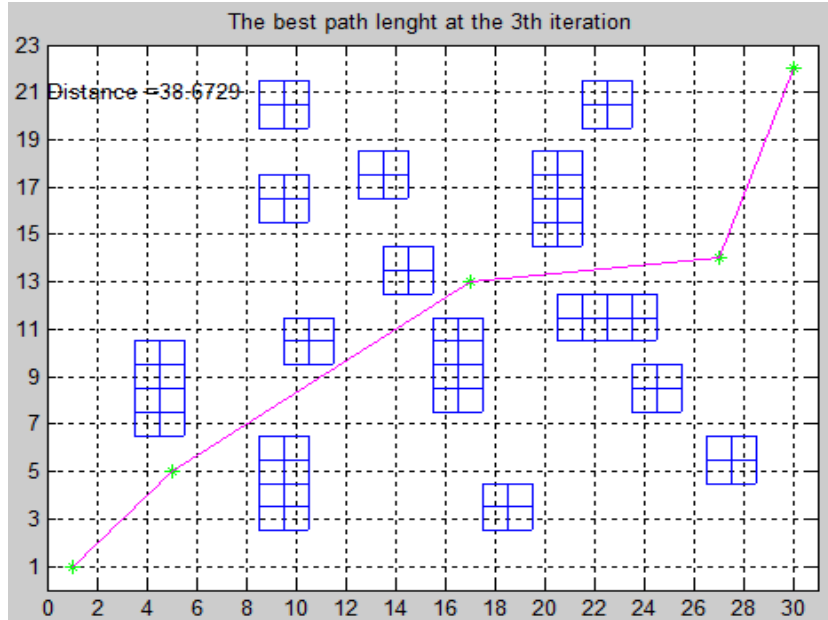

Fig 10: Path generated for the 1st environment using MGA* (3 iterations)

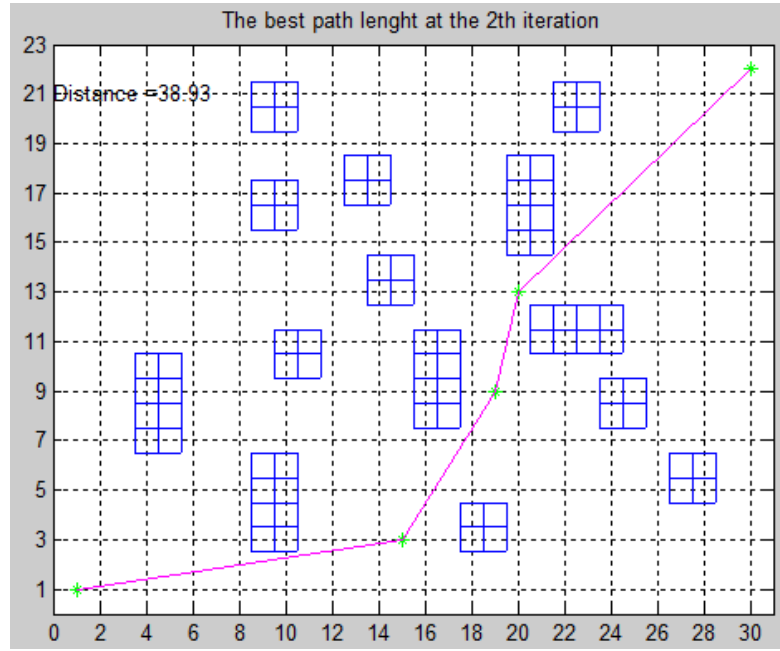

Fig 11: Path generated for the 1st environment using MGA* (2 iterations)

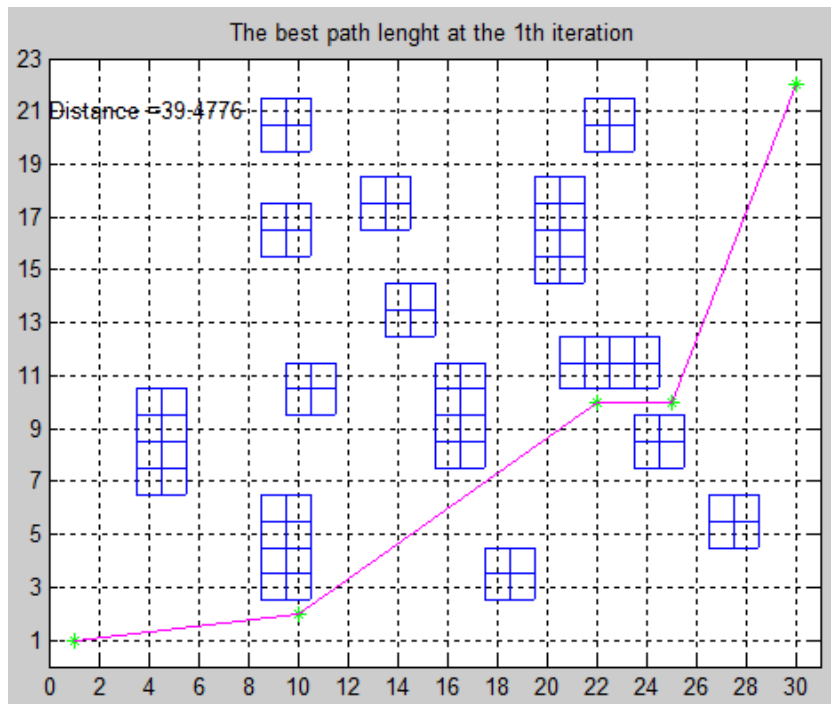

Fig 12: Path generated for the 1st environment using MGA* (1 iteration)

\subsubsection{Implementation of $M G A *$ for the Second} Environment

The performance of the $M G A^{*}$ is examined by applying a simulation for the $2^{\text {nd }}$ environment as illustrated in Figure 3 . The best simulations obtained by applying the $M G A^{*}$ to the $2^{\text {nd }}$ environment are shown in Table 2, and also the best path obtained is illustrated by Figure 13 and Figure 14.

Table 2: The simulation results for the 2th environment using MGA*

\begin{tabular}{|c|c|c|c|}
\hline \multicolumn{4}{|c|}{ Simulation Results } \\
\hline $\begin{array}{c}\text { Initial } \\
\text { Population }\end{array}$ & Iterations & Distance & Elapsed time [s] \\
\hline 100 & 2 & 19 & 67.76 \\
\hline 100 & 1 & 20.099 & 44.10 \\
\hline
\end{tabular}

Based on this result, it can be concluded that, the global optimal path obtained by the $M G A *$ is the optimal solution.

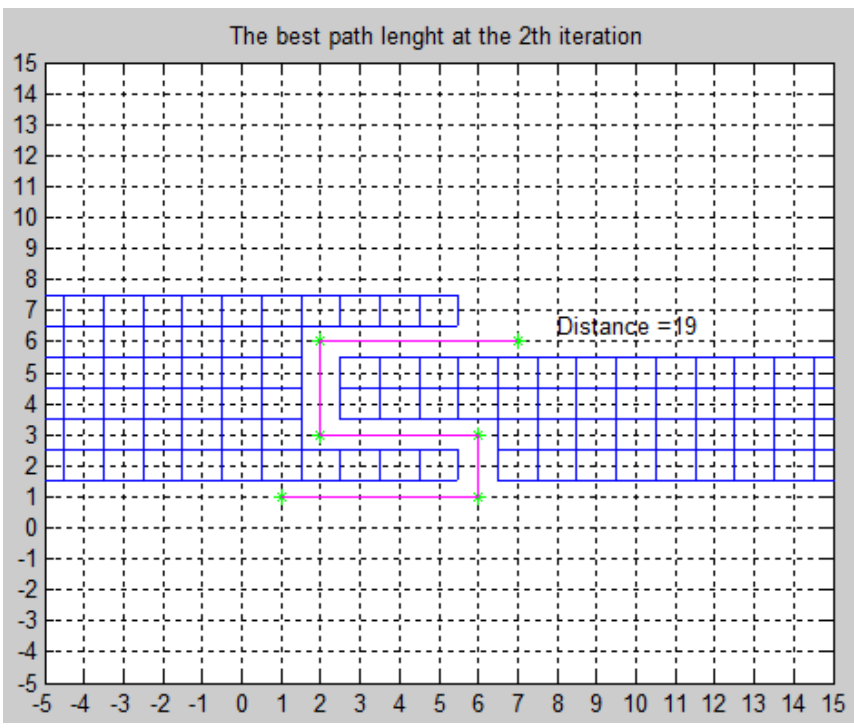

Fig 13: Path generated for the 2nd environment using MGA* (2 iterations)

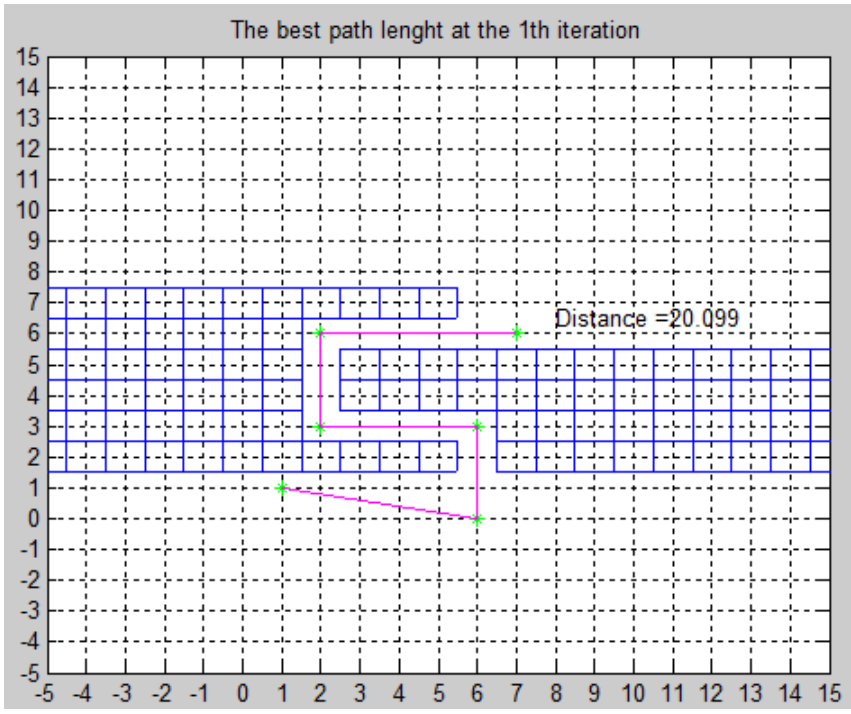

Fig 14: Path generated for the 2nd environment using MGA* (1 iteration) 


\subsubsection{Implementation of $M G A *$ for the Third Environment (Closed Environments)}

In order to show that the algorithm does not converge when there is no path to the target; the algorithm has been implemented to the two previous environments which turned to closed environments. Table 3 shows the elapsed time for the closed environments with different number of population size. For the environments the elapsed time is increased by increasing the number of population gradually because the large number of population requires larger computations.

Table 3: The simulation results for the $3^{\text {th }}$ environment using $M G A^{*}$

\begin{tabular}{|c|c|c|}
\hline $\begin{array}{c}\text { Maximum } \\
\text { population } \\
\text { size }\end{array}$ & $\begin{array}{c}1^{\text {st }} \text { Environment } \\
\text { elapsed time [s] }\end{array}$ & $\begin{array}{c}2^{\text {nd }} \text { Environment } \\
\text { elapsed time [s] }\end{array}$ \\
\hline 500 & 4.663363 & 0.390006 \\
\hline 1000 & 5.202287 & 0.560971 \\
\hline 2000 & 7.287891 & 1.357995 \\
\hline 5000 & 20.430164 & 6.895290 \\
\hline 10000 & 67.191626 & 26.559073 \\
\hline
\end{tabular}

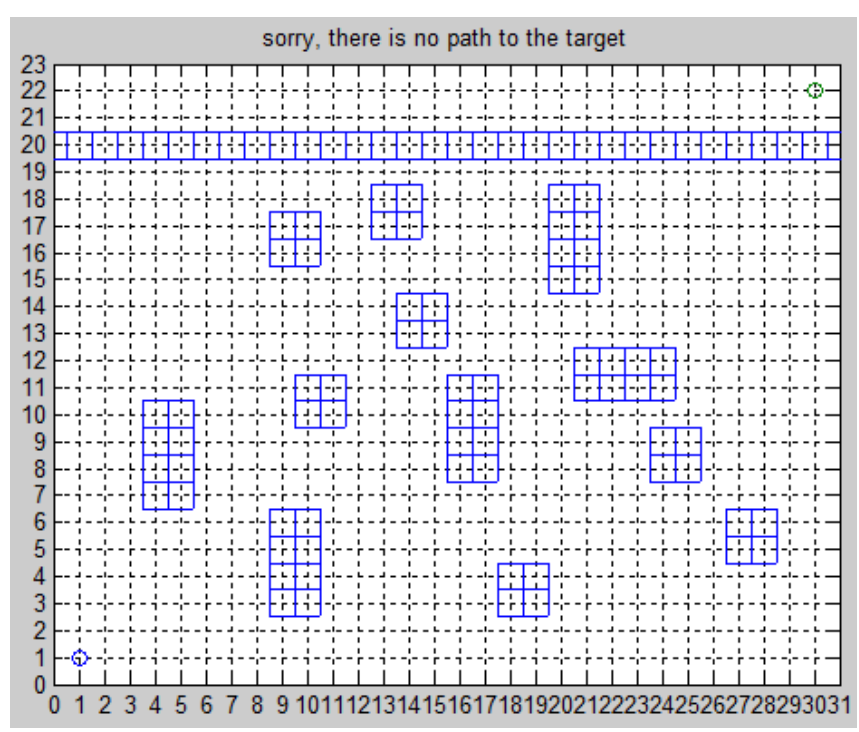

Fig 15: First closed environment using MGA*

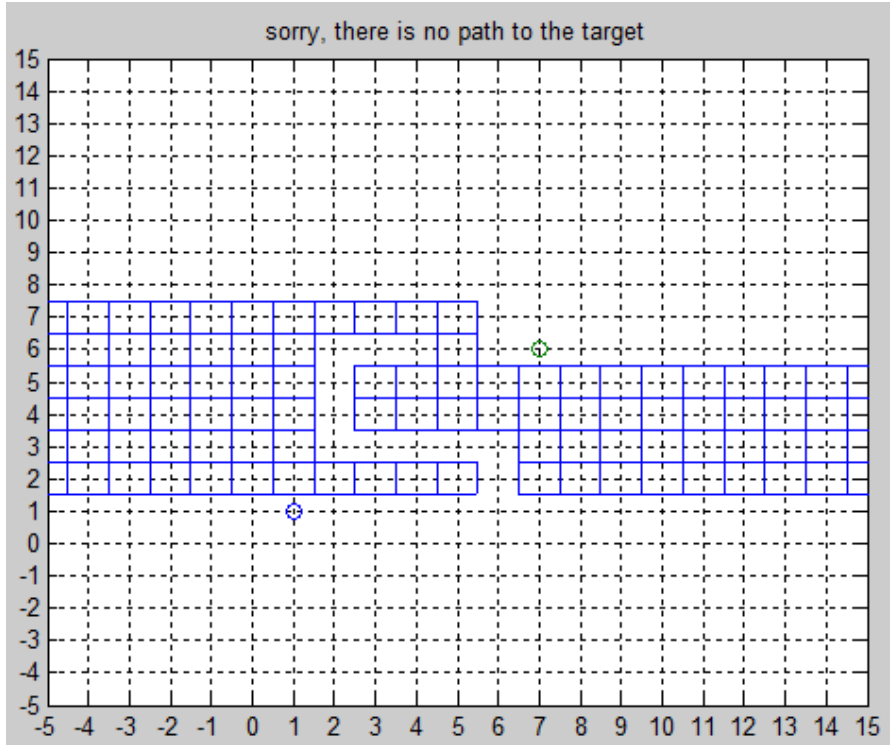

Fig 16: Second closed environment using MGA*

The simulation results shown in Figure 15 and Figure 16. The Figures show that if the $M G A^{*}$ could not find a path between the start point and the target point for the five working environments, an error message is returned so that the mobile robot could not find the target.

\subsection{The Implementation of Modified Particle Swarm Optimization (MPSO*) in Path Planning}

To study the performance of the MPSO*, the proposed algorithm have been applied to different types of working environments presented from Figure 2 to Figure 3. To investigate the effect of different swarm size on the performance of the MPSO*, different number of particles has been taking into account (i.e., 5, 10, 50, and 100). The particle's size has been selected on the base of trial and error, because there has been no recommendation in the literature regarding swarm size in PSO.

In the MPSO* various combinations of parameters were tested and the best obtained result are the values of weighing factors in fitness function $\mathrm{w}_{\mathrm{d}}=100$ and $w_{t}=1$, inertia weighing factor $w=0.02$. The swarms with small initial inertia weight converged relatively fast at the beginning of optimization. The chosen values of cognitive scaling and social scaling factors are $c_{1}=c_{2}=2$. Usually $c_{1}$ equals $c_{2}$ and ranges from 0 to 4 (Raja and Pugazhenthi, 2009).

\subsubsection{Implementation of MPSO* for the First Environment}

The performance of the MPSO* algorithm is examined by applying it to the $1^{\text {st }}$ environment shown in Figure 2 to find the optimal path. The best obtained simulation results with various numbers of particles, swarm size, for the $1^{\text {st }}$ environment is shown in Table 4. 
Table 5: The simulation results for the $1^{\text {st }}$ environment using $M P S O *$

\begin{tabular}{||c|c|c|c||}
\hline \multicolumn{4}{|c||}{ Simulation Results } \\
\hline $\begin{array}{l}\text { No. of } \\
\text { Particle }\end{array}$ & Iterations & Distance & Elapsed time [s] \\
\hline 5 & 33 & 39.4558 & 0.95 \\
\hline 10 & 31 & 39.4558 & 0.97 \\
\hline 50 & 30 & 39.4558 & 1.14 \\
\hline 100 & 31 & 39.4558 & 1.41 \\
\hline
\end{tabular}

The best solution obtained after running the MPSO* algorithm to this environment is shown in Figure 17 to Figure 20.

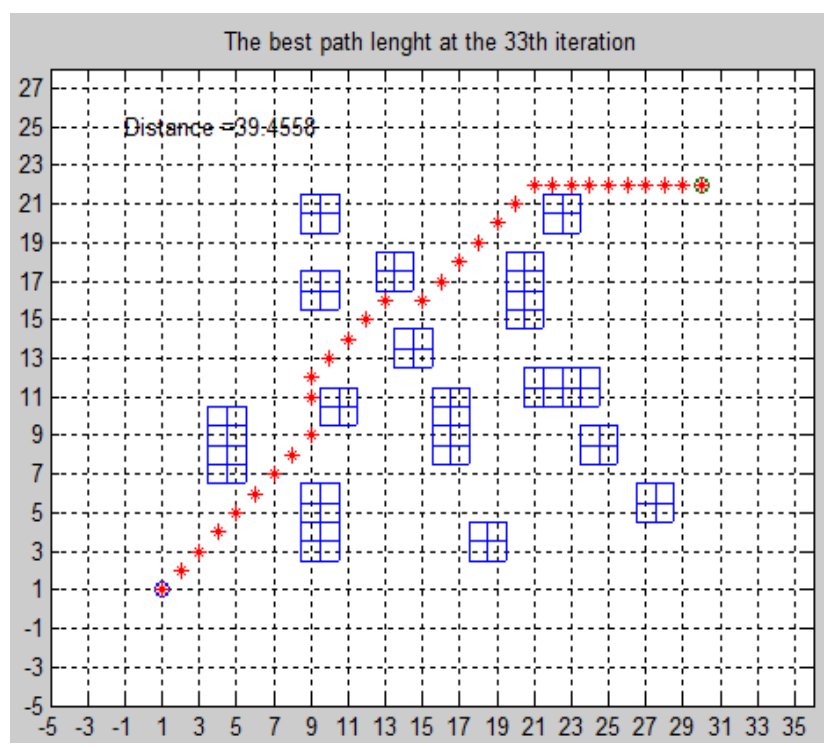

Fig 17: Path generated for the 1st environment using MPSO* (5 particles)

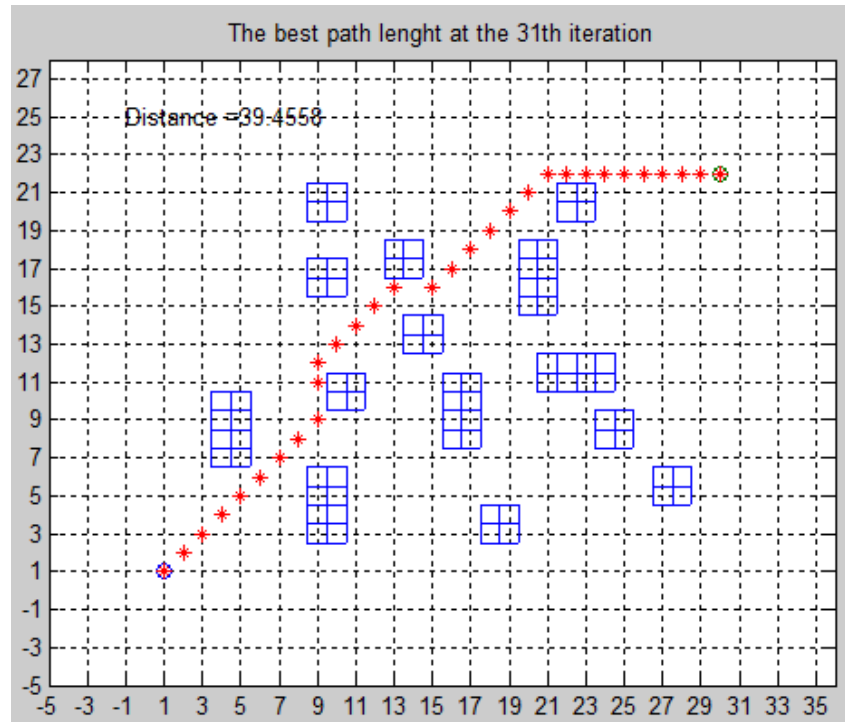

Fig 18: Path generated for the 1st environment using MPSO* (10 particles)

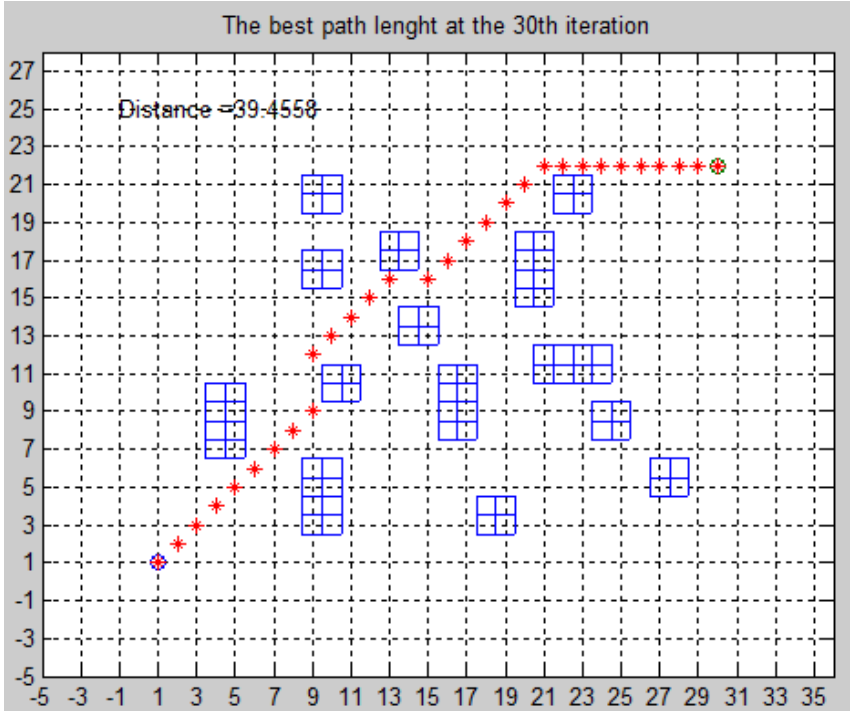

Fig 19: Path generated for the 1st environment using MPSO* (50 particles)

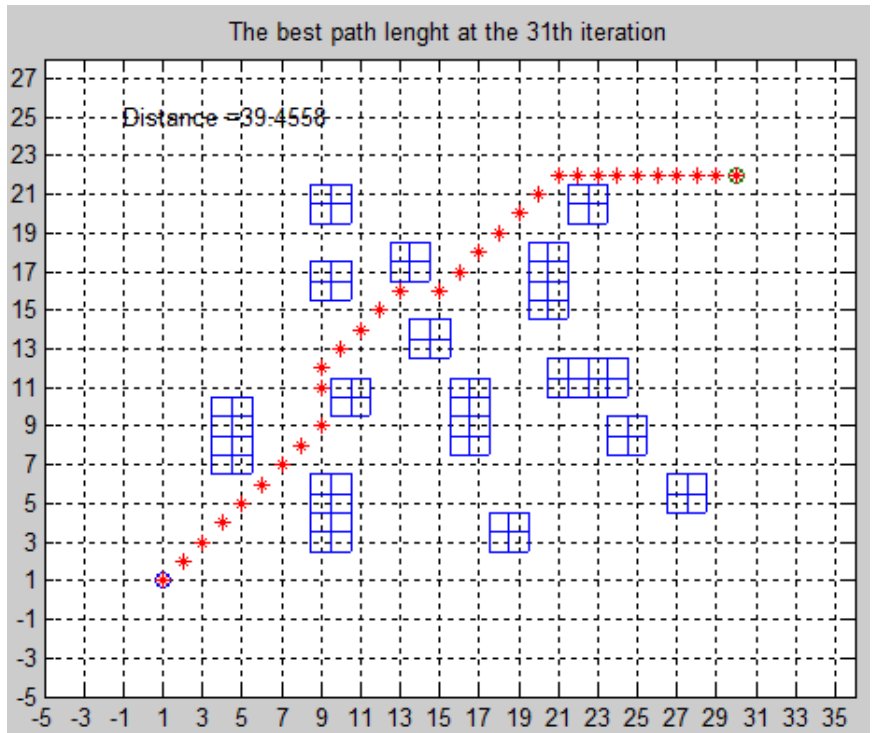

Fig 20: Path generated for the 1st environment using MPSO* (100 particles)

It can be observed from the above figures that no improvement is achieved for more than 5 particles in term of the path length; therefore 5 particles are sufficient to find the optimal path with length of 39.4558 in 0.95 second.

\subsubsection{Implementation of MPSO* for the Second Environment}

The capability of the $M P S O^{*}$ algorithm is examined by applying it to the $2^{\text {nd }}$ working environment, as shown in Figure 3, to find the optimal path with satisfied time. The simulation results obtained are shown in Table 5, and the results are demonstrated by Figure 21 to Figure 25 Fig. 
Table 5: The simulation results for the 2nd environment using MPSO*

\begin{tabular}{|c|c|c|c||}
\hline \multicolumn{4}{|c||}{ Simulation Results } \\
\hline $\begin{array}{l}\text { No. of } \\
\text { Particle }\end{array}$ & Iterations & Distance & Elapsed time [s] \\
\hline 2 & 26 & 27 & 1.19 \\
\hline 3 & 29 & 25 & 1.23 \\
\hline 5 & 20 & 23 & 1.25 \\
\hline 7 & 23 & 21 & 1.35 \\
\hline 10 & 20 & 19 & 1.41 \\
\hline
\end{tabular}

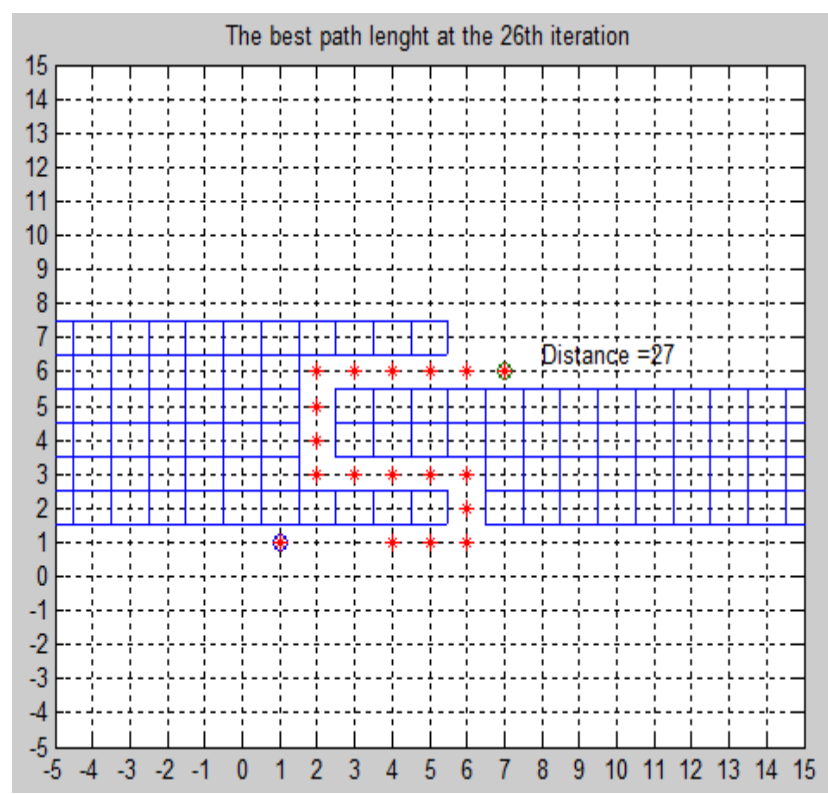

Fig 21: Path generated for the 2nd environment using MPSO* (2 particles)

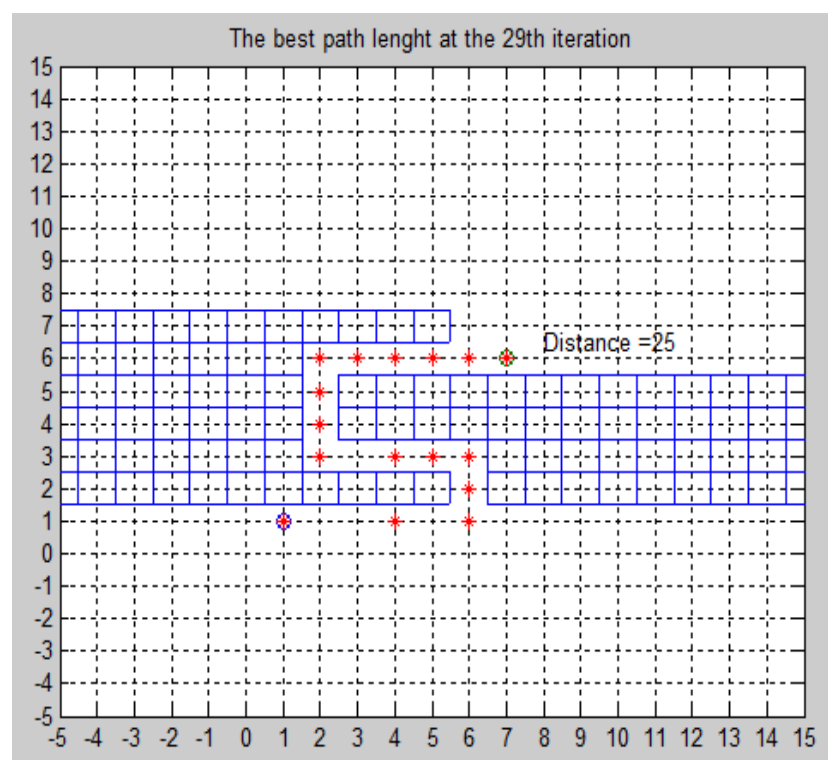

Fig 22: Path generated for the 2nd environment using MPSO* ( 3 particles)

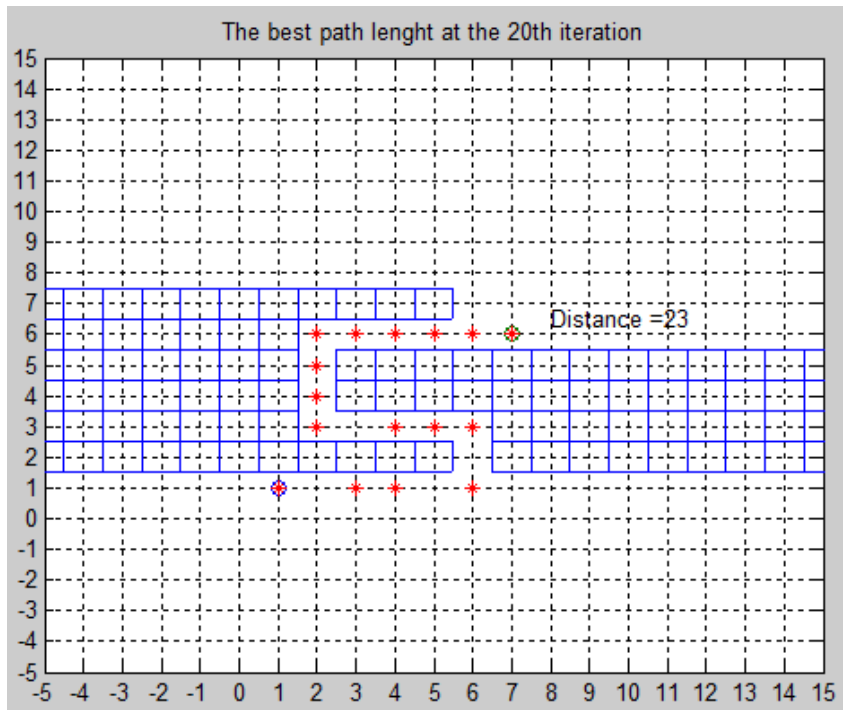

Fig 23: Path generated for the 2nd environment using MPSO* (5 particles)

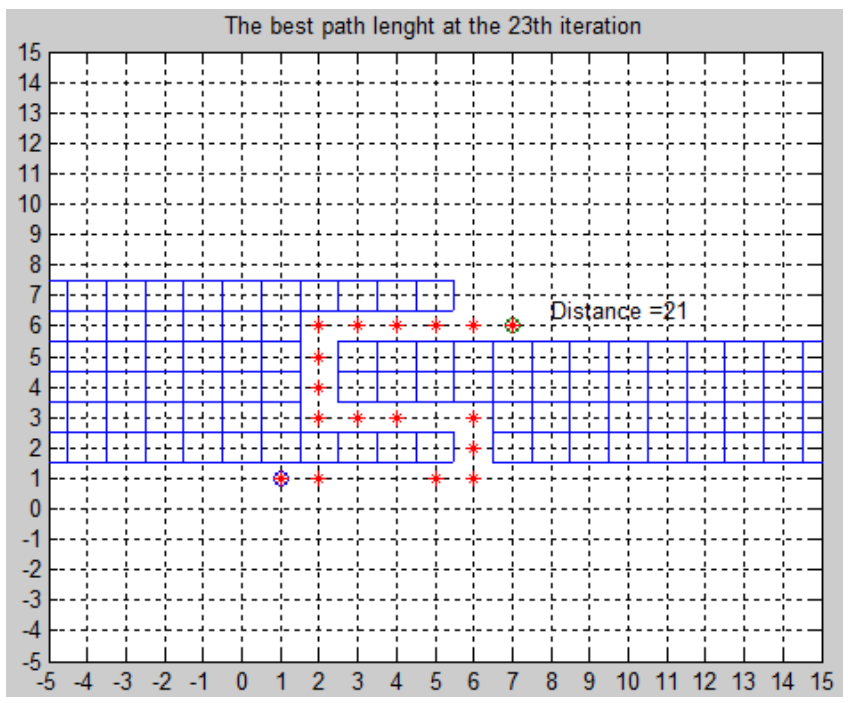

Fig 24: Path generated for the 2nd environment using MPSO* (7 particles)

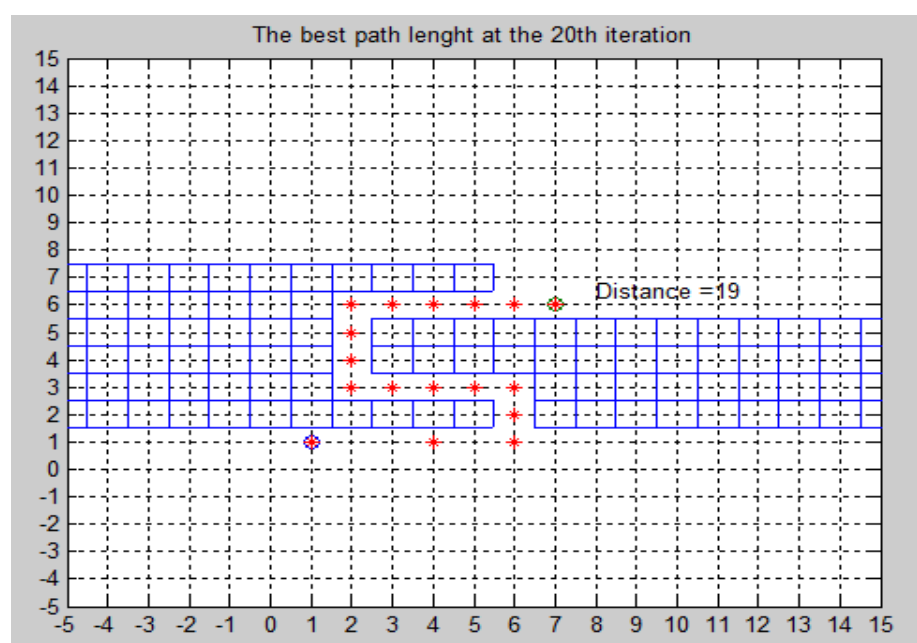

Fig 25: Path generated for the 2nd environment using MPSO* (10 particles) 
This algorithm has been examined with various swarm size to investigate the behavior of MPSO* algorithm in each case. This will also help in showing that $M P S O^{*}$ algorithm will converge to the optimal solution, optimal path, in each run. Through the simulation results, it is shown that the MPSO* can find out an optimal path very quickly.

\subsubsection{Implementation of MPSO* for the Third Environment (Closed Environments)}

The MPSO* algorithm has been carried out to the five previous environments which turned to closed environments, in order to demonstrate that the proposed algorithm does not converge when there is no path to the target Table 6. shows the elapsed time for the five closed environments with various number of particles and different number of iterations. As shown in the table, for all closed environments the elapsed time is increased by increasing the number of particles and the number of iterations because of that the large number of particle and iteration require larger computations.

Table 6: The simulation results for the 3rd environment using MPSO*

\begin{tabular}{|c|c|c|}
\hline No. of Particle & $\begin{array}{c}1^{\text {st }} \\
\text { Environment } \\
\text { Elapsed time } \\
{[\mathrm{sec}]}\end{array}$ & $\begin{array}{c}2^{\text {nd }} \\
\text { Environment } \\
\text { Elapsed time } \\
{[\mathrm{sec}]}\end{array}$ \\
\hline \multicolumn{3}{|c|}{10 iteration } \\
\hline 5 & 0.567463 & 0.348209 \\
\hline 10 & 0.624700 & 0.350721 \\
\hline 50 & 0.661831 & 0.674306 \\
\hline 100 & 0.682113 & 0.946107 \\
\hline \multicolumn{3}{|c|}{50 iteration } \\
\hline 5 & 0.639178 & 0.385743 \\
\hline 10 & 0.696363 & 0.578581 \\
\hline 50 & 1.223272 & 1.856159 \\
\hline 100 & 1.636615 & 2.946691 \\
\hline \multicolumn{3}{|c|}{100 iteration } \\
\hline 5 & 0.669309 & 0.460107 \\
\hline 10 & 0.753709 & 0.709233 \\
\hline 50 & 1.656838 & 2.655063 \\
\hline 100 & 2.893451 & 3.499064 \\
\hline
\end{tabular}

The results illustrated in Figure 26 to Figure 30 showed that if the proposed algorithm could not find a path between the start point and the target point for the five working environments an error message is returned. In all cases, the MPSO* algorithm succeeded to avoid the obstacle, even a collision free path did not exist in the closed environments.

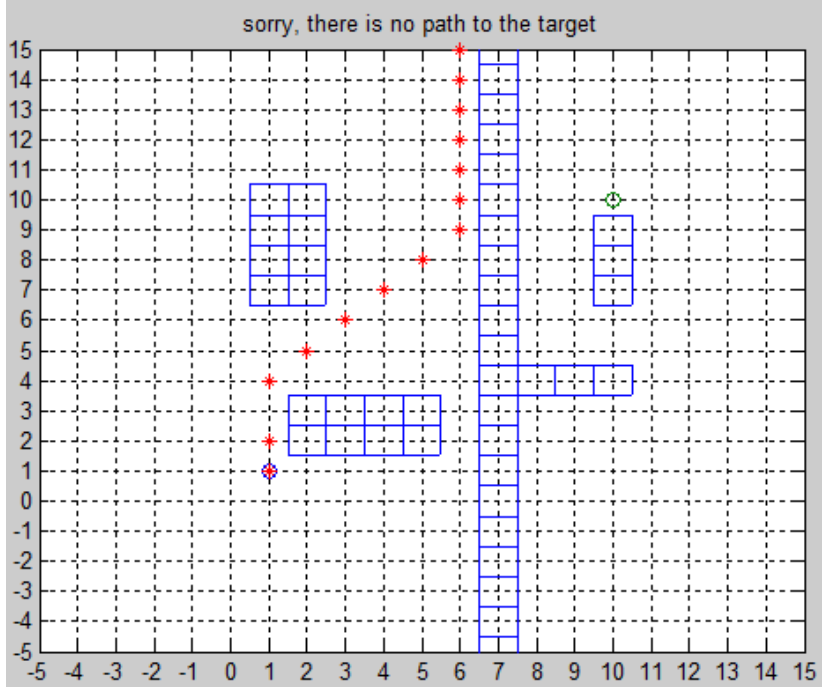

Fig 26: First closed environment using MPSO**

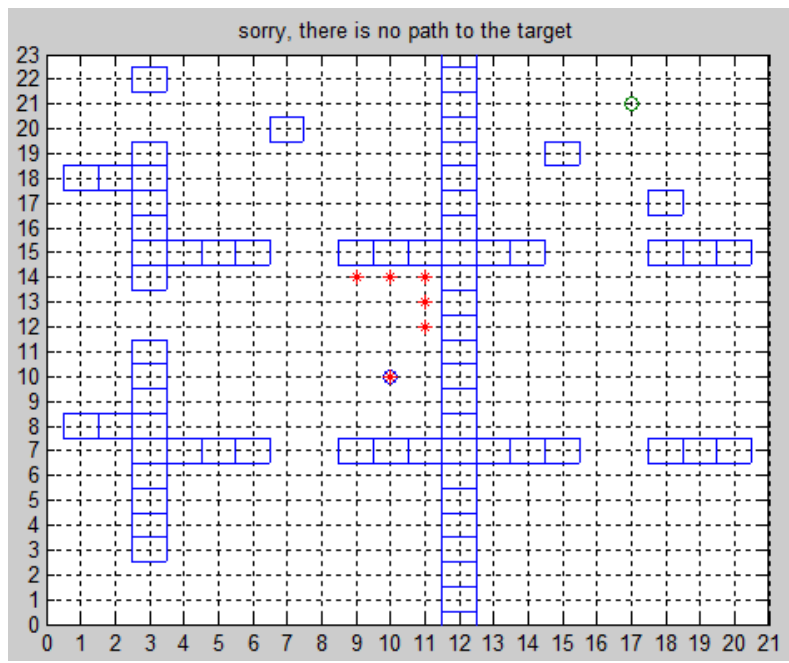

Fig 27: Second closed environment using MPSO*

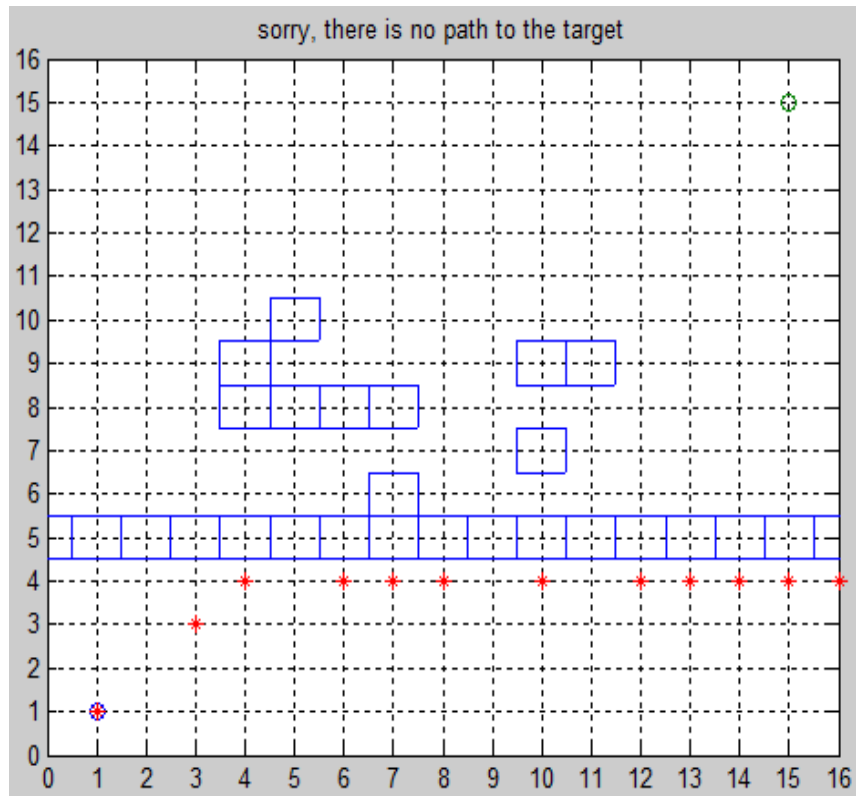

Fig 28: Third closed environment using MPSO* 


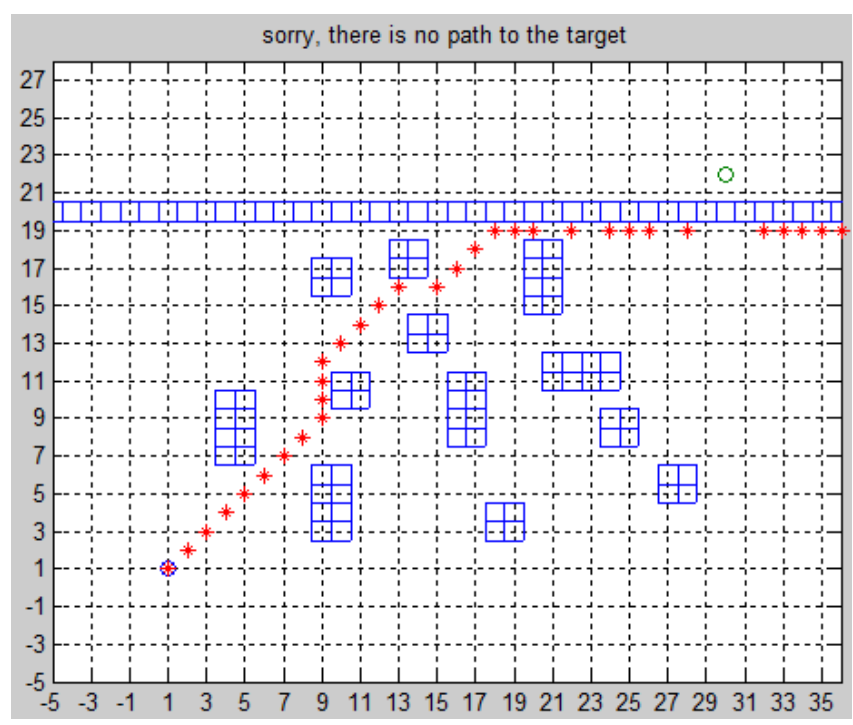

Fig 29: Fourth closed environment using MPSO*

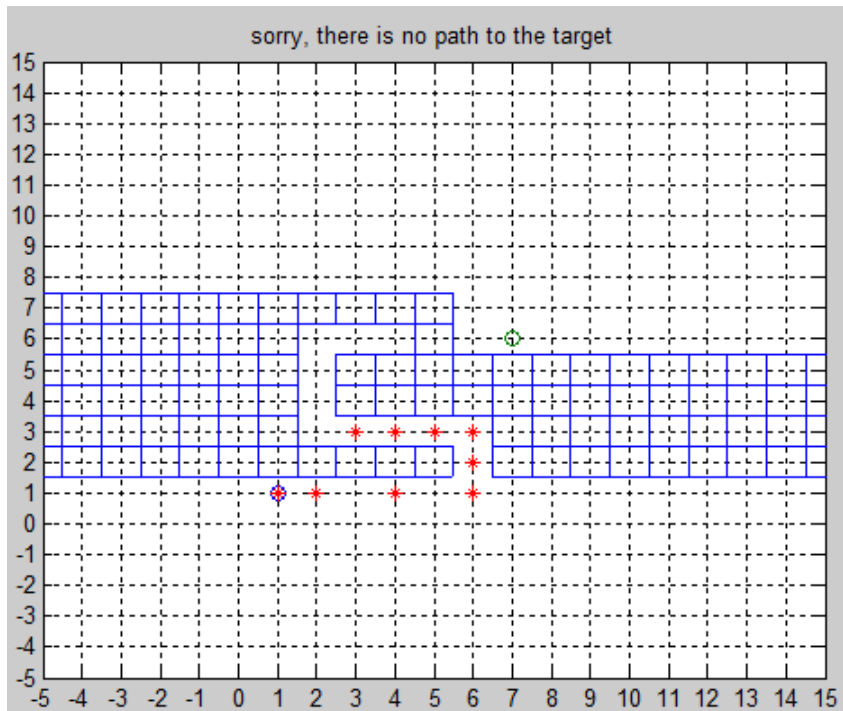

Fig 30: Fifth closed environment using MPSO*

\subsection{Comparative Study between MGA* and MPSO*}

This section describes comparison results between the $M G A^{*}$ and the MPSO*. The achieved simulation results of both algorithms for path length and travel time for the five working environments are shown in Table 7 and Table 8 , respectively. The results showed the performance of the proposed algorithms to find a feasible path for a mobile robot to move from a starting point to the target point in static environments. Results of these simulations are very encouraging and they indicate important contributions to the areas of path planning in mobile robots.

Table 7: Comparison of path length between MGA* and MPSO*

\begin{tabular}{|l|l|l||}
\hline \hline & $1^{\text {st }}$ Environment & $2^{\text {nd }}$ Environment \\
\hline MGA $^{*}$ & 37.9303 & 19 \\
\hline MPSO $^{*}$ & 39.4558 & 19 \\
\hline Diff $_{1}$ & 1.5255 & 0 \\
\hline
\end{tabular}

Diff $_{1}=$ path length $\left.{ }_{(M P S O *}\right)$ - path length ${ }_{\left(M G A^{*}\right)}$
Table 8: Comparison of calculation time [second] between MGA* and MPSO*

\begin{tabular}{|c|c|c|}
\hline & $4^{\text {th }}$ Environment & $5^{\text {th }}$ Environment \\
\hline $\mathrm{MGA}^{*}$ & 5.63 & 67.76 \\
\hline MPSO* & 0.95 & 1.41 \\
\hline Diff $_{2}$ & 4.68 & 66.35 \\
\hline
\end{tabular}

\section{CONCLUSIONS}

From the simulation results one can conclude that the proposed algorithms, MGA* and MPSO*, are capable of effectively guiding a robot moving from start position to the goal position in different working environments and find optimal/shortest path without colliding any obstacle in the environments. Also the simulation results show that the $\mathrm{MGA}^{*}$ and the MPSO* algorithms have the capability of finding global optimum path in complex environment which is clearly observed in the first environment. The using adaptive population size without selection and mutation operators in the proposed MGA* led to improve the execution time. Adaptive population size grows depending on the size, structure and the number of obstacles in the environment which led to improve the execution time. In the MPSO* an error factor is modeled to ensure that the PSO converges. These errors are used to change the particle's direction to head toward the target. A modified procedure carried out in the MPSO* to solve the infeasible path problem. When the particle path falls with an obstacle boundary, it is relocated to a position outside of the obstacle. The results obtained from the implementation of the MGA* and the MPSO* to find the optimal path for mobile robot show that the MGA* is more efficient than the MPSO* in term of minimizing distance, while the MPSO* is more efficient than the MGA* in terms of minimizing the execution time.The MGA* and the MPSO* algorithms didn't converge in the closed environments in which there is no path between the start point and the target, as observed in the closed environment. However, the MPSO* is faster to discover that the environment is closed. The MPSO* can rightfully be regarded as a good choice due to its convergence speed and robustness in global search

\section{REFERENCES}

[1] Patnaik, S., Jain, L. C., Tzafestas, S. G., Resconi, G., Konar, A. 2005 Innovations in Robot Mobility and Control, Springer, Netherlands.

[2] Kolski, S. 2007. Mobile Robots Perception and Navigation, Advanced Robotic Systems International and pro literatur Verlag, Germany.

[3] Han, K. M., 2007. Collision free path planning algorithms for robot navigation problem, Master Thesis, University of Missouri-Columbia.

[4] Yun, S.C., Ganapathy, V., Chong, L.O. 2010. Improved genetic algorithms based optimum path planning for mobile robot, International Conference on Control, Automation, Robotics and Vision, ICARCV, pp. 15651570. 
[5] Mohanty, P. K., and Parhi D. R. 2013, Controlling the Motion of an Autonomous Mobile Robot Using Various Techniques: a Review, Journal of Advance Mechanical Engineering, Vol. 1: pp.24-39.

[6] Dutta, S., 2010. Obstacle Avoidance of Mobile Robot using PSO-based Neuro Fuzzy Technique, International Journal of Computer Science and Engineering, Vol. 2, Issue, (2): pp. 301-304.

[7] Tamilselvi, D., shalinie, M., Hariharasudan. 2011. Optimal Path Selection for Mobile Robot Navigation Using Genetic Algorithm", International Journal of Computer Science Issues, Vol. 8, Issue, (4): pp.433-440.

[8] Ragavan, S.V., Ponnambalam, S.G., Sumero, C. 2011. Waypoint-based Path Planner for Mobile Robot Navigation Using PSO and GA-AIS", Recent Advances in Intelligent Computational Systems (RAICS), pp. 756-760

[9] Raja, P., Pugazhenthi, S. 2012 .Optimal path planning of mobile robots: A review, International Journal of Physical Sciences, Vol. 7, Issue, (9): pp. 1314 - 1320

[10] Ahmadzadeh, S., Ghanavati, M. 2012. Navigation of Mobile Robot Using the PSO Particle Swarm Optimization, Journal of Academic and Applied Studies (JAAS), Vol. 2, Issue, (1): pp. 32-38.
[11] Habeeb, Z. Q., 2012. A Simulation for Optimal Path Planning for Mobile Robot Using Modified Genetic Algorithm, Master Thesis, University of Baghdad.

[12] Yu-qin, W., Xiao-peng, Y. 2012. Research for the Robot Path Planning Control Strategy Based on the Immune Particle Swarm Optimization Algorithm, $2^{\text {nd }}$ International Conference on Intelligent System Design and Engineering Application, pp. 724-727.

[13] Gigras, Y., Gupta, K. 2012. Artificial Intelligence in Robot Path Planning", International Journal of Soft Computing and Engineering (IJSCE), Vol. 2, Issue, (2): pp.471-474.

[14] Jatmiko, W., Sekiyama K., and Fukuda, T. 2006. Modified Particle Swarm Robotic for Odor Source Localization in Dynamic Environment, the International Journal of Intelligent Control and Systems: Special Issue on Swarm Robotic, Vol. 11, Issue, (3): pp.176-184.

[15] Eberhart, R., and Shi, Y. 1998. Comparison between Genetic Algorithms and Particle Swarm Optimization. In Proceedings of the Seventh Annual Conference on Evolutionary Programming, Springer-Verlag, pp. 611619. 